\title{
Avaliação da implantação do Programa de Valorização do Profissional da Atenção Básica no estado da Bahia
}

\author{
Evaluation of the implementation of the Primary Care Professional \\ Valorization Program in the state of Bahia
}

Anderson Freitas de Santana', Ana Luiza Queiroz Vilasbôas²

DOI: 10.1590/0103-11042018S214

RESUMO O Programa de Valorização do Profissional da Atenção Básica foi criado no Brasil, em 2011, para apoiar a atuação de profissionais de saúde, sob supervisão, em municípios com carência de profissionais. Este estudo avaliou o grau de implantação do programa no estado da Bahia. Foram realizadas entrevistas semiestruturadas com membros da Comissão Coordenadora Estadual do programa e utilizadas fontes documentais para a produção dos dados. Os elementos críticos do processo de implantação foram analisados mediante o uso do triângulo de governo proposto por Matus. O programa, no estado da Bahia, alcançou 82,39\% do grau de implantação de acordo com o modelo lógico elaborado a partir das normativas federais. O grupo de gestão executou as atividades do programa com êxito, em função de sua capacidade de governo e do apoio político e administrativo da Secretaria Estadual de Saúde, ainda que não tenha havido incremento de recursos financeiros para potencializar os componentes do programa. Evidenciou-se o envolvimento dos atores da coordenação da supervisão e da supervisão médica na tentativa de reorientar a formação médica, apostando em dispositivos pedagógicos e outros recursos educacionais.

PALAVRAS-CHAVE Avaliação em saúde. Atenção Primária à Saúde. Programas governamentais. Médicos de atenção primária. Recursos humanos em saúde.

1 Universidade Federal da Bahia (UFBA), Instituto de Saúde Coletiva (ISC) Salvador (BA), Brasil. Orcid: 0000-0002-28882426

andersonfreitas13@gmail. com

2 Universidade Federal da Bahia (UFBA), Instituto de Saúde Coletiva (ISC) Salvador (BA), Brasil. Orcid: 0000-0002-55668337 analuvilas@gmail.com
ABSTRACT The Primary Care Professional Valorization Program was created in Brazil, in 2011, to support the performance of health professionals, under supervision, in municipalities with a shortage of professionals. This study evaluated the degree of implementation of the program in the state of Bahia. Semi-structured interviews were conducted with members of the State Coordinating Committee of the program and documentary sources were used to produce the data. The critical elements of the implementation process were analyzed through the use of the governance triangle proposed by Matus. The program, in the state of Bahia, reached $84 \%$ of the degree of implementation according to the logical model elaborated from the federal regulations. The management group successfully implemented the program's activities, due to its governance capacity and the political and administrative support of the State Health Secretariat, although there has been no increase in financial resources to leverage the components of the program. 
It was evidenced the involvement of the actors of the coordination of medical supervision and supervision in an attempt to reorient medical training, betting on pedagogical devices and other educational resources.

KEYWORDS Health evaluation. Primary Health Care. Government programs. Physicians primary care. Health manpower.

\section{Introdução}

A análise da provisão de médicos para a Atenção Primária à Saúde tem sido debatida desde a década de 1960 e foi amplamente discutida na Conferência de Alma Alta, em 1978, apontando a necessidade do treinamento de médicos e a adoção de estratégias para incentivar profissionais de saúde a prestar serviços em áreas remotas e de difícil acesso em diversos sistemas de saúde e distintos contextos. No Brasil, essa responsabilidade foi reconhecida na Constituição Federal de 1988 e na Lei $n^{0} 8.080 / 1990$, pois, até então, não existia uma política governamental direcionada à formação de profissionais voltados para atender às necessidades do País, ${ }^{\mathbf{1 , 2}}$. Em 2011, o número de médicos nos países com sistemas universais de saúde era distinto do cenário brasileiro. Nesses países, a razão do número de médicos por 1.000 habitantes era superior a 2,36, enquanto no Brasil e, especificamente, na Bahia eram, respectivamente, 1,95 e 1,21, no mesmo período. Esses dados revelam que a má distribuição de médicos e a escassez desses profissionais tornam-se desafios para a implementação da Atenção Primária à Saúde 3,4.

A literatura internacional indica que as causas da escassez de médicos na Atenção Primária à Saúde estão relacionadas à formação inadequada dos profissionais, remuneração inferior a outras especialidades, distribuição desigual de profissionais entre os serviços de saúde, salários pouco atrativos, profissionais sem treinamento para atuar no nível de atenção primária, migração para outras regiões e países, inadequação da infraestrutura de unidades de saúde, precarização das relações de trabalho ${ }^{5-7}$.

Diversos estudos apontam que os gestores municipais têm enfrentado a má distribuição de médicos formulando estratégias para incentivar a ocupação dos postos de trabalho na Atenção Primária à Saúde. Contudo, a escassez de médicos parece ser mais bem enfrentada pelo nível central dos sistemas de saúde com a implementação de estratégias e incentivos monetários e não monetários por meio dos programas governamentais $\mathbf{6 , 8 , 9}$.

Para minorar essa problemática, muitos países têm adotado incentivos monetários e não monetários, a exemplo da interiorização dos cursos de graduação em medicina e de programas de residência médica, remuneração adequada, oferta de condições satisfatórias de moradia e infraestrutura na zona rural, regulação da distribuição de médicos por região de saúde, incentivos financeiros acrescidos aos salários, redução da dívida estudantil e bônus na nota final dos processos seletivos para acesso aos programas de residência médica ${ }^{5,6,10-16}$.

No Brasil, diversas estratégias foram criadas para prover profissionais médicos para a Atenção Primária à Saúde desde a década de 1960, com o Projeto Rondon e o Programa de Interiorização das Ações 
de Saúde e Saneamento (Piass), durante o regime militar. Seguiu-se com a criação do Programa de Interiorização do Sistema Único de Saúde (Pisus), em 1993, e do Programa de Interiorização do Trabalho em Saúde (Pits), em 2001. Também foram desenvolvidas estratégias visando a mudanças na formação médica, a exemplo do Programa de Incentivo a Mudanças Curriculares no Curso de Medicina (Promed) e do Programa Nacional de Reorientação da Formação Profissional em Saúde, em 2007, que foram interrompidos posteriormente por mudanças governamentais $\mathbf{1}^{1,7}$.

Em 2011, o governo brasileiro instituiu o Programa de Valorização do Profissional da Atenção Básica (Provab), por meio da Portaria Interministerial $\mathrm{n}^{\circ}$ 2.087, cujo fundamento era apoiar e incentivar médicos, enfermeiros e cirurgiões-dentistas para atuação na atenção básica de municípios com carência de profissionais, em áreas de extrema pobreza e nas periferias das regiões metropolitanas sob supervisão presencial e à distância ${ }^{15}$.

A revisão de literatura realizada na base de dados da Biblioteca Virtual em Saúde (BVS), entre os anos de 2014 e 2017, evidenciou a carência de estudos que avaliem o Provab no âmbito estadual, que buscassem identificar fatores contextuais relacionados à sua implantação. Assim, o objetivo deste estudo foi avaliar o grau de implantação do Provab no estado da Bahia, entre os anos de 2013 e 2015, buscando analisar aspectos favoráveis e obstáculos ao desenvolvimento do programa.

Sendo assim, as questões que nortearam a pesquisa foram as seguintes: qual foi o grau de implantação alcançado pelo Provab no estado da Bahia? Que fatores impulsionaram ou obstaculizaram a implantação do Provab nesse estado?

\section{Material e métodos}

Realizou-se pesquisa avaliativa, com abordagem estruturada na díade 'estrutura-processo'. Elegeu-se o componente $1 \mathrm{~b}$ da Análise de Implantação ${ }^{18}$, que corresponde à estimativa do grau de implantação da intervenção estudada.

Para fundamentar a produção dos dados empíricos da investigação, foi elaborado um modelo lógico preliminar do Provab a partir de fontes documentais. O modelo foi organizado em três componentes: condições funcionais e de trabalho, aspectos pedagógicos e gestão do programa. Essa versão do modelo lógico e a respectiva matriz de aferição dos critérios avaliativos foram submetidas a uma técnica de consenso simplificada, com a participação de cinco especialistas em atenção básica e formação médica. Após duas rodadas, os itens divergentes foram retirados do modelo lógico e da matriz de aferição dos critérios avaliativos. As respostas foram processadas no Microsoft Office Excel e tratadas estatisticamente através do cálculo de medidas de tendência central e dispersão, o que resultou no modelo lógico e na matriz de aferição dos critérios avaliativos agrupados segundo os componentes do programa (figura 1 e tabela 1). 
Figura 1. Modelo lógico do Programa de Valorização do Profissional da Atenção Básica, estado da Bahia, 2013 a 2015

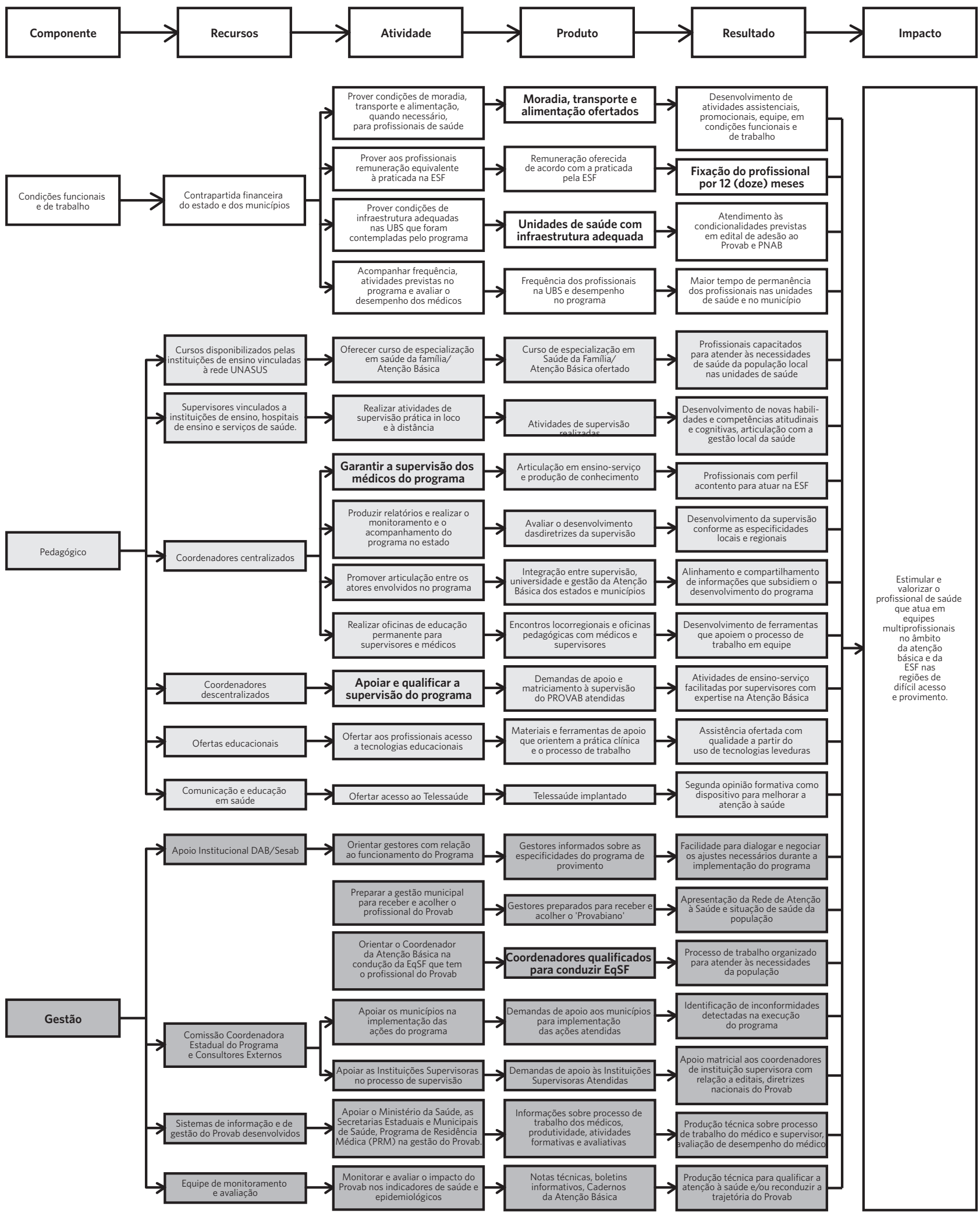

Fonte: Elaboração própria a partir de revisão documental. 
Para obtenção do percentual de implantação de cada componente do programa, utilizou-se o seguinte cálculo:

\section{Escore final $=$ Pontuação obtida $\quad$ x 100, Pontuação máxima}

Em que a pontuação obtida é o somatório da pontuação alcançada em cada critério e respectivo componente; enquanto a pontuação máxima é o somatório da pontuação máxima de cada critério e respectivo componente.

O somatório máximo de todos os componentes totalizou 159 pontos. Para estimar a implantação do Provab na Bahia, foram considerados os seguintes intervalos: de zero até 52 pontos ( $\geq 0$ e $\leq 33,3 \%)$ : não implantado; de 53 até 105 pontos (>33\% e $\leq 66,6 \%$ ): parcialmente implantado; de 106 pontos até 159 pontos (> 66\%): implantado.

Tabela 1. Matriz de critérios, indicadores e padrões para estimar o grau de implantação do Provab na Bahia

\begin{tabular}{|c|c|c|c|}
\hline & Critério & Padrão & $\begin{array}{r}\text { Pontuação } \\
\text { máxima }\end{array}$ \\
\hline & $\begin{array}{l}\text { Condições adequadas de moradia, transporte e alimenta- } \\
\text { ção, quando necessário }\end{array}$ & $\begin{array}{l}100 \% \text { das condições de moradia, transporte e alimenta- } \\
\text { ção adequadas, quando necessário }\end{array}$ & 09 \\
\hline & $\begin{array}{l}\text { Remuneração dos profissionais conforme os valores } \\
\text { praticados com os trabalhadores de outros vínculos }\end{array}$ & $\begin{array}{l}100 \% \text { dos profissionais do município contratados pelo } \\
\text { Provab na modalidade trabalhador-estudante }\end{array}$ & 09 \\
\hline & $\begin{array}{l}\text { Condições de infraestrutura (segurança, higiene, equipa- } \\
\text { mentos, insumos, instalações sanitárias) nas unidades } \\
\text { de saúde contempladas pelo programa }\end{array}$ & $\begin{array}{l}100 \% \text { das unidades de saúde adequadas para receber } \\
\text { profissional do Provab }\end{array}$ & 08 \\
\hline & $\begin{array}{l}\text { Cumprimento de carga horária de atividades assisten- } \\
\text { ciais nas unidades de saúde }\end{array}$ & 32 horas semanais & 09 \\
\hline & Total do componente & & 35 \\
\hline \multirow{9}{*}{$\begin{array}{l}\text { Componente } \\
\text { Condições } \\
\text { Funcionais de } \\
\text { Trabalho }\end{array}$} & $\begin{array}{l}\text { Oferta de curso de especialização no formato EAD (Edu- } \\
\text { cação a Distância) em saúde da família/atenção básica }\end{array}$ & $100 \%$ de médicos ativos matriculados & 09 \\
\hline & Equipe de supervisão médica & 1 supervisor/10 médicos & 09 \\
\hline & Elaboração de relatórios sobre a supervisão & 1 relatório/ano & 09 \\
\hline & Realização de Encontros Locorregionais (ELR) & 1 ELR/ano & 09 \\
\hline & Realização de oficinas de qualificação da supervisão & 1 oficina/ano & 09 \\
\hline & Apoio à supervisão médica & $\begin{array}{l}\text { 100\% de supervisores apoiados pelos coordenadores } \\
\text { descentralizados }\end{array}$ & 09 \\
\hline & Acesso ao Portal Saúde Baseada em Evidências & $\begin{array}{l}\text { 100\% dos profissionais com acesso ao Portal Saúde } \\
\text { Baseada em Evidências }\end{array}$ & 08 \\
\hline & Acesso ao Telessaúde & 100\% de profissionais com acesso ao Telessaúde & 08 \\
\hline & Total do componente & & 70 \\
\hline \multirow[t]{4}{*}{ Gestão } & $\begin{array}{l}\text { Orientação aos gestores municipais sobre o funciona- } \\
\text { mento do programa }\end{array}$ & $100 \%$ orientados sobre o funcionamento do programa & 09 \\
\hline & $\begin{array}{l}\text { Apoio institucional aos municípios partícipes do pro- } \\
\text { grama }\end{array}$ & 100\% de municípios participantes apoiados & 09 \\
\hline & $\begin{array}{l}\text { Orientação aos coordenadores de atenção básica sobre } \\
\text { o processo de trabalho do médico e supervisor }\end{array}$ & 100\% de coordenadores de atenção básica orientados & 09 \\
\hline & Apoio aos municípios na implementação do Provab & $100 \%$ das demandas de apoio atendidas & 09 \\
\hline
\end{tabular}


Tabela 1. (cont.)

Utilização dos dados disponíveis nos sistemas de infor-

100\% de relatórios analisados

As variáveis do triângulo de governo proposto por Matus ${ }^{19}$ foram utilizadas para caracterizar possíveis elementos favoráveis e restritivos à implantação do Provab, relacionados ao contexto político-institucional estadual, quais sejam: o projeto e a capacidade de governo e a governabilidade da equipe responsável pela execução do programa entre os anos de 2013 e 2015.

A Bahia foi selecionada intencionalmente por ser um dos estados do Brasil que teve o maior número de participantes no Provab entre 2013 e 2015. Localizado na Região Nordeste, possui uma área de $564.732,642 \mathrm{~km}^{2}$ e representa o quinto estado em extensão territorial. A população estimada, em 2015, correspondia a 15.276.566 habitantes ${ }^{20}$. A cobertura da Estratégia Saúde da Família (ESF) nesse período variou de $61,75 \%$ a $69,77 \% \mathbf{2 1}$.

Os dados do estudo foram obtidos mediante a realização de entrevistas semiestruturadas com informantes-chave e análise de documentos oficiais. Os entrevistados deveriam ser membros da Comissão Coordenadora Estadual (CCE) do Provab, com, pelo menos, um ano de atuação no programa. A amostra intencional de sujeitos do estudo foi condicionada pelo grau de saturação das informações obtidas nas entrevistas. No total, foram entrevistados oito informantes-chave envolvidos diretamente com a execução do Provab na Bahia. As entrevistas foram realizadas a partir de roteiro semiestruturado baseado no modelo lógico e nas variáveis do triângulo de governo, registradas através de gravador de áudio. Os documentos oficiais - a saber, atas de reuniões, manuais técnicos, editais e portarias - foram submetidos à análise de conteúdo ${ }^{22}$.

O projeto de pesquisa foi aprovado pelo Comitê de Ética em Pesquisa do Instituto de Saúde Coletiva da Universidade Federal da Bahia, sob parecer $n^{0}$ 2.011.684. A gestão estadual da atenção básica autorizou a realização da pesquisa mediante assinatura do Termo de Anuência Institucional. Todos os entrevistados assinaram o Termo de Consentimento Livre e Esclarecido (TCLE).

\section{Resultados}

\section{Grau de implantação do Provab no estado da Bahia}

O Provab no estado da Bahia, entre os anos 2013 e 2015, alcançou o percentual de 82,39\% de implantação. Evidenciou-se que o componente condições funcionais e de trabalho alcançou 88,57\% de implantação; em seguida, o componente pedagógico obteve $84,28 \%$ de implantação; e o componente de gestão atingiu $75,92 \%$ de implantação (tabela 2 ). 
Tabela 2. Grau de implantação do Provab segundo componente do modelo lógico, estado da Bahia, 2013 a 2015

\begin{tabular}{|c|c|c|c|c|}
\hline Componente & Critério & $\begin{array}{r}\text { Pontuação } \\
\text { máxima }\end{array}$ & $\begin{array}{r}\text { Pontuação } \\
\text { obtida }\end{array}$ & \% implantação \\
\hline \multirow{5}{*}{$\begin{array}{l}\text { Condições } \\
\text { funcionais e de } \\
\text { trabalho }\end{array}$} & Condições adequadas de moradia, transporte e alimentação, quando necessário & 09 & 08 & $88,8 \%$ \\
\hline & $\begin{array}{l}\text { Remuneração dos profissionais conforme os valores praticados com os trabalhado- } \\
\text { res de outros vínculos }\end{array}$ & 09 & 09 & $100 \%$ \\
\hline & $\begin{array}{l}\text { Condições de infraestrutura (segurança, higiene, equipamentos, insumos, instala- } \\
\text { ções sanitárias) nas unidades de saúde contempladas pelo programa }\end{array}$ & 08 & 06 & $75 \%$ \\
\hline & Cumprimento de carga horária de atividades assistenciais nas unidades de saúde & 09 & 08 & $88,8 \%$ \\
\hline & Total do componente & 35 & 31 & $88,57 \%$ \\
\hline \multirow[t]{9}{*}{ Pedagógico } & $\begin{array}{l}\text { Oferta de curso de especialização no formato Educação a Distância (EAD) em } \\
\text { saúde da família/atenção básica }\end{array}$ & 09 & 09 & $100 \%$ \\
\hline & Equipe de supervisão médica & 09 & 09 & $100 \%$ \\
\hline & Elaboração de relatórios sobre a supervisão & 09 & 06 & $66,66 \%$ \\
\hline & Realização de encontros locorregionais & 09 & 05 & $55,55 \%$ \\
\hline & Realização de oficinas de qualificação da supervisão & 09 & 09 & $100 \%$ \\
\hline & Apoio à supervisão médica & 09 & 09 & $100 \%$ \\
\hline & Acesso ao Portal Saúde Baseada em Evidências & 08 & 08 & $100 \%$ \\
\hline & Acesso ao Telessaúde & 08 & 04 & $50 \%$ \\
\hline & Total do componente & 70 & 59 & $84,28 \%$ \\
\hline \multirow[t]{8}{*}{ Gestão } & Orientação aos gestores municipais sobre o funcionamento do programa & 09 & 09 & $100 \%$ \\
\hline & Apoio institucional aos municípios partícipes do programa & 09 & 09 & $100 \%$ \\
\hline & $\begin{array}{l}\text { Orientação aos coordenadores de atenção básica sobre o processo de trabalho do } \\
\text { médico e supervisor }\end{array}$ & 09 & 09 & $100 \%$ \\
\hline & Apoio aos municípios na implementação do Provab & 09 & 09 & $100 \%$ \\
\hline & $\begin{array}{l}\text { Utilização dos dados disponíveis nos sistemas de informação do programa para } \\
\text { implementação do programa }\end{array}$ & 09 & 05 & $55,55 \%$ \\
\hline & $\begin{array}{l}\text { Utilização dos dados disponíveis nos sistemas de informação do programa para } \\
\text { analisar os indicadores de saúde e epidemiológicos nas equipes de saúde com } \\
\text { médicos do programa }\end{array}$ & 09 & 00 & $0 \%$ \\
\hline & Total do componente & 54 & 41 & $75,92 \%$ \\
\hline & TOTAL GERAL & 159 & 41 & $82,39 \%$ \\
\hline
\end{tabular}

A orientação dos gestores municipais quanto aos aspectos normativos do Provab foi efetivada, principalmente, pelos atores da Comissão Coordenadora Estadual e pelo apoio institucional da Diretoria de Atenção Básica (DAB), nos espaços de governança estadual e regional e nas visitas in loco nos municípios. A participação do apoio institucional foi essencial para orientar a (re) organização do processo de trabalho das equipes, de modo que as inconformidades e demandas identificadas no âmbito da operacionalização do Provab fossem encaminhadas à CCE para providências.

A Secretaria de Estado da Saúde da Bahia incorporou o programa na estrutura da DAB, colocando a organização administrativa à disposição dos atores do Provab. As principais atividades de monitoramento foram referentes ao cumprimento de condicionalidades e responsabilidades pelas gestões municipais, regularidade das informações nos 
sistemas de informação da atenção básica e assiduidade dos profissionais, realizadas através dos instrumentos da supervisão médica e dos sistemas de informação do Provab.

A Comissão Coordenadora Estadual atuava nas situações de descumprimento de condicionalidades e em outras inconformidades identificadas, instruindo processos e utilizando os instrumentos jurídicos e administrativos necessários até a resolução das inconformidades ou o arquivamento dos processos por outros desfechos. A capilaridade do apoio institucional nos territórios deu celeridade aos processos instruídos pela Comissão, e, quando necessário, eram elaborados Planos de Ação para que o gestor municipal assumisse a responsabilidade por regularizar as inconformidades.

Com relação à utilização de dados dos sistemas de informação do Provab para analisar os indicadores de saúde e epidemiológicos decorrentes do trabalho dos médicos do programa, não foram identificadas evidências que permitissem verificar a realização da atividade. Nesse sentido, essa lacuna pode ter comprometido a elaboração e a implementação de ações para responder às necessidades de saúde da população adstrita à unidade.

Os entrevistados destacaram a importância da Escola de Saúde Pública da Bahia na articulação e na participação nas atividades de organização e implementação do curso de especialização.

Ressalta-se a elaboração de um plano de trabalho com o propósito de alinhar e orientar o processo de trabalho da supervisão médica, bem como subsidiar elementos para reconduzir o trabalho da supervisão. Além disso, a coordenação da supervisão realizou diversas reuniões presenciais para acompanhamento e avaliação das atividades pedagógicas desenvolvidas.

Foram relatadas pelos entrevistados as principais facilidades para o desenvolvimento pedagógico, destacando-se: interação dos professores do curso de especialização ofertado à época pelo Instituto de Saúde Coletiva da Universidade Federal da Bahia
(UFBA); coordenação da supervisão engajada com a atenção básica, formação médica; supervisores com experiência em processos educativos. Também foram evidenciadas dificuldades no acompanhamento pedagógico relacionadas à ausência de um projeto político-pedagógico no âmbito da coordenação nacional; resistência dos médicos às atividades pedagógicas, visto que alguns consideravam o Provab como 'trampolim' para residência médica; alguns supervisores possuíam formação em áreas que não eram prioritárias para a atenção básica; alguns gestores municipais não investiam na integração ensino-serviço, e a educação permanente dependia do interesse do gestor - não era institucionalizada.

A composição da coordenação da supervisão do Provab na Bahia tinha uma característica singular em relação aos demais estados da federação. A equipe de coordenação da supervisão era formada por médicos de família e comunidade, pediatra, fisioterapeuta, enfermeiro e psicólogo, que, juntos, construíram uma proposta pedagógica consistente, com diferencial na organização tanto no âmbito da gestão quanto no pedagógico.

A equipe de supervisão, por sua vez, foi formada por médicos que tinham especializações em diversas áreas, em maior número nas áreas básicas (medicina de família e comunidade, pediatria, ginecologia e obstetrícia) e em menor quantitativo nas especialidades (oftalmologia, otorrinolaringologia, ortopedia, psiquiatria, anestesiologia etc). Ainda que o grupo condutor priorizasse especialidades das áreas da atenção básica, não havia candidatos selecionados em número suficiente.

Foi evidenciado que era disponibilizado para os supervisores material didático sobre gestão da clínica, gestão do cuidado, projeto terapêutico singular, agenda compartilhada, entre outras ferramentas da medicina de família e comunidade, de modo que o processo de trabalho dos supervisores estivesse alinhado e em conformidade com o plano de trabalho da supervisão. 


\section{Triângulo de governo: aspectos crí- ticos da implantação do Provab na Bahia}

As três variáveis que compõem o triângulo de governo, a saber: projeto e capacidade de governo e governabilidade, são construtos potentes para caracterizar aspectos do contexto político-institucional, que podem facilitar ou criar obstáculos para a implantação de uma intervenção. No caso particular do Provab, na Bahia, os achados deste estudo indicam que o sucesso na implantação do programa, evidenciado pelo grau de implantação superior a $80 \%$, estava relacionado com a 'força' dessas três variáveis. Havia um projeto de governo compartilhado entre os atores responsáveis pela operacionalização do programa, elevada capacidade de governo e autonomia administrativa das estruturas da Secretaria Estadual de Saúde (Sesab), apoio político do Ministério da Saúde, Conselho Estadual de Secretários Municipais de Saúde (Cosems), e dos municípios ao provimento médico pelo Provab.

\section{Projeto de governo}

Quanto ao projeto de governo, a Sesab apostou no Provab como um meio de dar respostas às necessidades dos municípios quanto ao provimento de profissionais para atenção básica, podendo ser evidenciado pelo compromisso do secretário estadual da saúde, que defendia a qualificação da atenção básica, a ampliação do acesso, a reorientação do modelo de atenção, a formação profissional ordenada pelo Sistema Único de Saúde (SUS). De igual modo, a direção da Faculdade de Medicina (Fameb) da UFBA entendia que o Provab seria o propulsor de transformações do curso de graduação em Medicina.

As evidências apontaram que a CCE do Provab tinha clareza da finalidade do programa, e suas atividades apontavam que a imagem-objetivo estava centrada na formação médica na atenção básica. A pauta do Provab foi considerada prioritária no colegiado executivo da DAB. Foi considerado como projeto estratégico para qualificar a atenção básica, sendo incorporado no processo de trabalho dos técnicos dessa diretoria.

\section{Governabilidade}

A Sesab dispunha de apoio político à implantação do Provab na Bahia, que era evidenciado, entre outros fatores, pelo suporte da direção da Fameb e do Instituto de Saúde Coletiva, unidades acadêmicas da UFBA que tinham expertise na formação médica e em pesquisa em saúde coletiva. Havia, também, o apoio da Escola de Saúde Pública da Bahia. Destaque-se que o Gabinete do Secretário foi apoiado pela direção da Fameb no debate com a corporação médica sobre o Provab.

Existiu um trabalho intenso entre DAB e Gabinete do Secretário de saúde, com análise política e estratégica dos municípios potenciais para implantação do programa. Todos os pedidos que eram solicitados ao secretário eram prontamente respondidos de modo que o programa pudesse funcionar a contento.

Os entrevistados apontaram que a Sesab disponibilizou recursos materiais e humanos na estrutura da DAB, com ofertas de tecnologias de monitoramento e avaliação, assim como as Diretorias Regionais de Saúde (Dires) contribuíram com a logística para o desenvolvimento das atividades de supervisão médica, educação permanente para os membros do grupo condutor e qualificação do corpo de supervisão médica.

Embora a Bahia tenha priorizado a implantação do Provab à época, não investiu recursos financeiros diretamente no programa. Investiu indiretamente através das atividades do apoio institucional que foram essenciais para efetivar a interlocução entre a CCE e os municípios. A insuficiência de recursos financeiros dificultou a realização de oficinas de qualificação dos médicos e viagens para acompanhamento dos supervisores médicos. 
Por sua vez, a extinção das Dires, em dezembro de 2014, fragilizou a relação do programa com os municípios, pois elas faziam a interlocução com estes. Por outro lado, o bom relacionamento existente entre a DAB e o Departamento de Atenção Básica do Ministério da Saúde pode ser atribuído ao fato de o governo do estado da Bahia e o governo federal serem ligados ao mesmo partido político.

Quanto à participação do Cosems, as evidências apontam que essa entidade teve atuação favorável à implantação do Provab no tocante ao provimento, orientando os gestores com relação à provisão de médicos, mas não tinha clareza sobre os outros componentes do Provab. Contudo, o apoio dos gestores municipais foi importante para a implantação do Provab. Apesar do número elevado de gestores interessados na adesão ao programa, nem todos garantiram estrutura adequada para acolher o médico do Provab e proporcionar uma vivência qualificada e exitosa nas unidades de saúde.

\section{Capacidade de governo}

O secretário estadual de saúde, à época da implantação do Provab, dispunha de formação, experiência política e técnica, o que foi essencial para firmar o compromisso da instituição com o programa. Por sua vez, a capacidade técnica dos coordenadores das instituições supervisoras contribuiu para a implantação do Provab na Bahia. Os profissionais que integravam a coordenação das instituições supervisoras do Provab tinham formação em saúde coletiva, no mínimo, especialização em saúde coletiva, e alguns tinham mestrado. A liderança exercida pelo grupo condutor pode ser constatada pelo protagonismo e pela autonomia da CCE, o que permitiu ampliar a composição da comissão, com a inserção de uma representação dos médicos do Provab; e a composição da coordenação da supervisão, com a inclusão de atribuições administrativas e pedagógicas.

As evidências apontam que a seleção de supervisores médicos para o programa com formação na saúde coletiva ou em medicina de família e comunidade era um desafio a ser superado, já que não existiam muitos médicos com os requisitos e a experiência desejada. Não era uma diretriz nacional ter supervisores com esse perfil, mas o estado da Bahia optou por ter médicos supervisores com formação e experiência em saúde coletiva, atenção básica, medicina de família e áreas afins, docência e integração ensino-serviço.

Embora fosse realizado o monitoramento do Provab na Bahia, não foi construído um instrumento capaz de avaliar o programa em todos os seus componentes. Também não aconteceu no âmbito nacional uma avaliação que contemplasse todas as dimensões do Provab e que apontasse as fragilidades, potencialidades e os desafios.

Destaque-se que a organização do trabalho foi sendo construída no decorrer da implantação do Provab e à medida que iam surgindo as necessidades nos territórios apoiados, o que indica que a equipe do CCE foi ampliando sua capacidade de governo ao longo do desenvolvimento do programa.

\section{Discussão}

Esta pesquisa, ao avaliar o grau de implantação do Provab no estado da Bahia, identificou fragilidades e dificuldades na implantação do programa, que teve como finalidade enfrentar a escassez de profissionais médicos no SUS naquele território. Seus achados podem contribuir para o aperfeiçoamento de estratégias de provisão de médicos no Brasil.

A CCE do Provab foi essencial no processo de orientação dos elementos normativos do programa aos gestores municipais e no acolhimento e na orientação dos profissionais médicos. $\mathrm{O}$ engajamento dos atores que atuam no âmbito da gestão estadual é fundamental para o êxito da implantação de políticas de saúde ${ }^{23}$. 
A participação do apoio institucional da Diretoria de Atenção Básica Estadual foi considerada primordial para orientar, divulgar e acompanhar o Provab nos municípios participantes do programa. Estudos apontam que o apoio institucional tem sido efetivo para qualificar a gestão municipal no que tange à implantação de projetos estratégicos na atenção básica. Tem sido necessária na orientação dos trabalhadores sobre o funcionamento dos programas de provimento e, principalmente, na articulação dos supervisores com gestores municipais para a execução das atividades do componente pedagógico

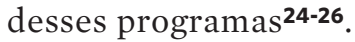

Neste estudo, não foi observada qualquer evidência que permita inferir a utilização dos dados dos sistemas de informação do Provab para analisar indicadores de saúde e epidemiológicos da população adstrita às Equipes de Saúde da Família (EqSF) em que atuavam médicos do Provab. A análise dos dados permitiria inferências sobre o trabalho dos profissionais nos programas de provimento e das suas respectivas equipes, principalmente com relação à ampliação do acesso à saúde e ao comportamento dos indicadores de internação por causas sensíveis à atenção básica ${ }^{25,27,28}$.

As atividades de monitoramento foram imprescindíveis para a identificação de inconformidades, especialmente na oferta de condições de infraestrutura das unidades de saúde contempladas pelo programa. Estudos apontam que a inadequação de infraestrutura de unidades de saúde pode comprometer o desenvolvimento de atividades assistenciais e, no caso particular do Provab, obstaculizar a formação dos profissionais e ser determinante para o desligamento de profissionais ${ }^{29,30}$.

Destaca-se a importância de o município possuir infraestrutura das unidades de saúde adequadas e existência de materiais para ampliar o escopo de prática dos profissionais participantes dos programas de provimento. Em outro estudo, evidenciou-se que a distância entre as unidades e o local de residência dos profissionais, assim como a indisponibilidade de materiais para realização do trabalho são fatores que apresentaram maior correlação com a rotatividade de profissionais médicos ${ }^{31,32}$.

A supervisão médica desempenhou as atividades administrativas e gerenciais a contento, resultando na redução expressiva do número de processos administrativos na CCE. Contudo, outros estudos apontam que o trabalho do supervisor médico deve estar pautado nas dimensões técnico-pedagógica e gerencial-administrativa e deve extrapolar os muros da supervisão tradicional, pois desse modo estimula o processo de aprendizagem ${ }^{25,33}$.

Sobre a assiduidade dos médicos, este estudo evidenciou que a ação de acompanhamento de carga horária dos profissionais foi crucial para garantir a permanência do profissional na unidade de saúde e ampliar o acesso. Diversos estudos apontam que a assiduidade dos médicos na atenção básica amplia o acesso aos serviços ofertados pela equipe de saúde e melhora a satisfação dos usuários. A implantação de programas de provimento registra maior permanência do médico na unidade de saúde e tem impactos significativos sobre os indicadores de saúde ${ }^{34,35}$.

A supervisão do Provab na Bahia não se assemelhava a uma supervisão tradicional, que é compreendida como o exercício de uma função gerencial com capacidade de comando para execução de atividades técnico-administrativas. Foi concebida a partir da perspectiva do apoio institucional e tem como pilar o componente pedagógico capaz de construir junto com o outro e ofertar ferramentas para o desenvolvimento das ações ${ }^{24,36}$.

A ausência de um projeto político-pedagógico no âmbito nacional e o descompromisso de alguns gestores municipais foram evidenciados como alguns dos fatores dificultadores para o desenvolvimento das atividades da supervisão. O projeto político-pedagógico vai expressar as atividades pedagógicas e 
didáticas que levam a instituição formadora a alcançar os objetivos traçados. A ausência do projeto político-pedagógico no processo educacional pode conduzir a diversas trajetórias e dificultar a organização do trabalho dos supervisores ${ }^{29}$.

O objetivo do Provab estava explicitado nos marcos legais e nas falas institucionais dos atores do Ministério da Saúde. O discurso e o posicionamento da Comissão Coordenadora Estadual para investir na formação dos médicos eram ratificados através das diversas estratégias educacionais implementadas. As evidências encontradas neste estudo apontam que o estado da Bahia desejava produzir mudanças na formação médica e, consequentemente, na reorganização do processo de trabalho da equipe de atenção básica.

O processo de implantação do Provab no âmbito da Sesab foi facilitado pela capacidade do gestor estadual de mobilizar diretorias e trabalhadores e por ter colocado à disposição da CCE a estrutura física da secretaria, os recursos humanos e as Dires. Sem estrutura física e sem equipamentos adequados, a gestão tem sua capacidade de governo enfraquecida e limitada, na medida em que os gestores que são colocados nessas condições interferem na capacidade de resposta resolutiva do grupo ${ }^{37}$.

A elevada capacidade de governo do secretário estadual foi fundamental para a implantação do Provab. A participação de um ator de alta direção da instituição dotado de capital cognitivo acerca da temática é determinante para configuração do projeto de governo e para a boa governabilidade. As instituições com menor grau de implantação das ações intersetoriais foram aquelas em que os sujeitos tinham governabilidade limitada, porém, o sujeito com governabilidade dentro das suas instituições favorece a operacionalização das ações ${ }^{\mathbf{3 8}, 39}$.

O alinhamento entre a Diretoria de Atenção Básica Estadual e o Departamento de Atenção Básica do Ministério da Saúde foi de suma importância na realização de proposições para o Provab. Uma boa relação entre setores, instituições e instâncias de gestão favorece a implantação de intervenções ${ }^{38}$.

Mostrou-se eficaz a atuação do grupo condutor do Provab e do apoio institucional na garantia das condicionalidades relacionadas à oferta adequada de unidades de saúde, com infraestrutura das unidades e composição da EqSF. Descentralizar o poder decisório permite que cada nível gerencial se ocupe dos problemas que são de maior magnitude ${ }^{40}$.

\section{Considerações finais}

O presente estudo reforça a ideia de que o trabalho integrado de atores governamentais na implantação de uma intervenção proporciona alcançar os objetivos desejados. A Comissão Coordenadora Estadual apresentou uma robusta capacidade de governo que pode ter contribuído para a implementação do Provab segundo o projeto de garantia de provimento médico como estratégia de qualificação da atenção básica na Bahia, apoiado pelo Gabinete do Secretário.

Aponta-se a necessidade de uma maior participação dos atores governamentais na mobilização dos gestores municipais quanto à priorização das atividades pedagógicas nas estratégias de provisão de médicos.

Embora a supervisão seja um dispositivo potente no processo formativo dos médicos, também deve ser de responsabilidade dos atores que conduzem a Política de Atenção à Saúde no âmbito municipal.

A atual conjuntura político-econômica do Brasil ameaça a sustentabilidade e a consolidação do SUS. Presencia-se o enfraquecimento e a desestruturação de políticas e programas estruturantes da atenção básica, a exemplo do Provab e do Programa Mais Médicos.

Cabe destacar que esse estudo se limitou a avaliar o grau de implantação do Provab. Não teve como objetivo avaliar a influência 
da interação entre o contexto de implantação e a intervenção sobre os efeitos observados, sendo necessária a realização de novos estudos de modo que seja possível compreender a variação desses efeitos pós-implantação do Provab e de outros programas de provimento semelhante a ele.

\section{Referências}

1. Maciel Filho R. Estratégias Para a Distribuição e Fixação de Médicos em Sistemas Nacionais de Saúde: O Caso Brasileiro [tese]. Rio de Janeiro: Universidade Estadual do Rio de Janeiro; 2007. 264 p.

2. Brasil. Fundo das Nações Unidas para a Infância. Alma Ata 1978, Cuidados Primários de Saúde. Brasília, DF: Unicef; 1979.

3. Scheffer M, Biancarelli A, Cassenote A, coordenadores. Demografia Médica no Brasil: dados gerais e descrição das desigualdades. Brasília, DF: CFM; 2011.

4. Universidade Federal de Minas Gerais. Faculdade de Medicina. Núcleo de Estudos em Saúde Coletiva. Construção do índice de escassez de profissionais de saúde para apoio à Política Nacional de Promoção da Segurança Assistencial em Saúde. Belo Horizonte: UFMG; 2010.

5. Shtasel D, Hobbs-Knutson K, Tolpin H, et al. Developing a Pipeline for the Community-Based Prima-

\section{Colaboradores}

Santana AF e Vilasbôas ALQ participaram da concepção, delineamento, análise e interpretação dos dados; redação, revisão crítica do artigo e aprovação da versão a ser publicada. ry Care Workforce and Its Leadership: The Kraft Center for Community Health Leadership's Fellowship and Practitioner Programs. Acad Med. 2015 set; 90(9):1272-1277.

6. Nkomazana O, Mash R, Shaibu S, et al. Stakeholders' Perceptions on Shortage of Healthcare Workers in Primary Healthcare in Botswana: Focus Group Discussions. Plos One. 2015 ago; 10(8):1-15.

7. Talib ZM, Baingana RK, Sagay AS, et al. Investing in community-based education to improve the quality, quantity, and retention of physicians in three African countries. Educ Health (Abingdon). 2013; 26(2):109-114.

8. Salsberg ES. Is the Physician Shortage Real? Implications for the Recommendations of the Institute of Medicine Committee on the Governance and Financing of Graduate Medical Education. Acad Med. 2015 set; 90(9):1210-1214.

9. Holte JH, Kjaer T, Abelsen B, et al. The impact of 
pecuniary and non-pecuniary incentives for attracting young doctors to rural general practice. Soc Sci and Med. 2015 mar; 128:1-9.

10. Ozegowski S. Effective policy mechanisms for an equitable geographical distribution of general practitioners: a qualitative comparative analysis of the accessibility of primary care in Europe. J Health Serv Res Policy. 2013 jul; 18(3):151-159.

11. Silva RB; Pineault R. Impact of physician distribution policies on primary care practices in rural Quebec. Can J Rural Med. 2012; 17(3):92-98.

12. Cooper JK, Heald K, Samuels M. Affecting the supply of rural physicians. Am J Public Health. 1977 ago; 67(8):756-759.

13. Berger CB, Dallegrave D, Castro Filho ED, et al. A formação na modalidade Residência Médica: contribuições para a qualificação e provimento médico no Brasil. Rev Bras Med Fam Comunidade. 2017 jan-dez; 2(39):1-10.

14. Cameron IAN. Increasing recruitment and retention of doctors in rural and remote areas: Australia as a case study. In: Anais do Seminário Internacional de Política de Recursos Humanos em Saúde. Brasília, DF: Ministério da Saúde; 2002. p. 45-52.

15. Brasil. Ministério da Saúde. Portaria Interministerial $\mathrm{n}^{\circ} 2.087$, de $1^{\circ}$ de setembro de 2011 . Institui o Programa de Valorização do Profissional da Atenção Básica. Diário Oficial da União. 15 Maio 2013.

16. Rabinowitz HK. Recruitment, retention, and follow-up of graduates of a program to increase the number of family physicians in rural and underserved areas. N Engl J Med. 1993; 328(13):934-939.

17. Carvalho MS. Programa de Valorização dos Profissionais da Atenção Básica: um olhar implicado sobre sua implantação [dissertação] [internet]. Brasília, DF: Universidade de Brasília; 2013 [acesso em 2015 jul 15]. 167 p. Disponível em: http://repositorio.unb.br/bitstream/10482/13660/1/2013_M\%C3 \%B4nicaSampaiodeCarvalho.pdf.
18. Champagne F, Brousselle A, Hartz Z, et al. A Análise da Implantação. In: Brousselle, A, Champagne F, Contandriopoulos AP, et al. Avaliação: conceitos e métodos. Rio de Janeiro: Fiocruz; 2011. p. 217-241.

19. Matus C. O Triângulo de Governo. In: Matus C. Política, Planejamento e Governo. Brasília, DF: Ipea; 1993. p. 59-62.

20. Instituto Brasileiro de Geografia e Estatística. Projeção da população do Brasil e das unidades da federação [internet]. Rio de Janeiro: IBGE; 2016 [acesso em 2016 set 15]. Disponível em: http://www.ibge. gov.br/apps/populacao/projecao/index.html.

21. Brasil. Ministério da Saúde. Proporção de cobertura estimada populacional de saúde da família [internet]. Brasília, DF: Ministério da Saúde; c2013-2015 [acesso em 2016 set 16]. Disponível em: https:// egestorab.saude.gov.br/paginas/acessoPublico/relatorios/relHistoricoCoberturaAB.xhtml.

22. Bardin L. Análise de Conteúdo. Portugal: Edições 70; 1977.

23. Lima LL, Ascenzi LD. Implementação de Políticas Públicas: Perspectivas Analíticas. Rev Sociol Polit. $2013 \mathrm{dez} ; 48(21): 101-110$.

24. Falleiro LM, Barros RS, Lima VCZ, et al. Considerações preliminares sobre Apoio Institucional e Educação Permanente. In: Falleiro LM, organizadora. Experiências de Apoio Institucional no SUS: da teoria à prática. Porto Alegre: Rede Unida; 2014. p. 1930.

25. Silva KCS. Processo de Trabalho do Médico no Programa de Valorização Profissional da Atenção Básica - PROVAB: Bahia: é difícil 'quebrar' essa caixinha focada na doença [dissertação]. Feira de Santana: Universidade Estadual de Feira de Santana; 2016. 149 p.

26. Vieira MC. Avaliação das atividades de supervisão nos programas de provimento de médicos na Bahia [dissertação] [internet]. Campinas: Universidade Estadual de Campinas; 2017 [acesso em 2017 maio 
14]. 128 p. Disponível em: http://repositorio.unicamp.br/bitstream/REPOSIP/322468/1/Vieira MariangelaCosta_MP.pdf.

27. Lima EJF, Santana DDB, Lima LCS, et al. Como os Egressos do Provab e Aprovados na Residência Avaliaram a Experiência? Rev Bras Educ Méd. 2016; 40(4):731-738.

28. Miranda AS, Melo DA. Análise comparativa sobre a implantação do Programa Mais Médicos em agregados de municípios do Rio Grande do Sul, Brasil. Ciênc Saúde Colet. 2016; 21(9):2837-2848.

29. Nóbrega-Therrien SM, Guerreiro MGS, Moreira TMM, et al. Projeto Político Pedagógico: concepção, construção e avaliação na enfermagem. Rev Esc Enferm USP. 2010; 44(3):679-686.

30. Gonçalves RF, Sousa IMC, Tanaka OY, et al. Programa Mais Médicos no Nordeste: avaliação das internações por condições sensíveis à Atenção Primária à Saúde. Ciênc Saúde Colet. 2016; 21(9):2815-2824.

31. Girardi SN, Carvalho CL, Pierantoni CR, et al. Avaliação do escopo de prática de médicos participantes do Programa Mais Médicos e fatores associados. Ciênc Saúde Colet 2016; 21(9):2739-2748.

32. Campos CVA, Malik AM. Satisfação no trabalho e rotatividade dos médicos do Programa de Saúde da Família. Rev Adm Pública. 2008 mar-abr; 42(2):347368.

33. Botti SHO, Rego S. Preceptor, Supervisor, Tutor e Mentor: Quais são Seus Papéis? Rev Bras Educ Méd. 2008; 32(3):363-373.

34. Campos RTO, Ferrer AL, Gama CAP, et al. Avaliação da qualidade do acesso na atenção primária de uma grande cidade brasileira na perspectiva dos usuários. Saúde debate. 2014; 38(esp):252-264.

35. Organização Pan-Americana da Saúde. $160^{a}$ Sesión del Comité Ejecutivo: Estrategia de Recursos Humanos para el Acceso Universal a La Salud y la Cobertura Universal de Salud. Washington, DC: Opas; 2017.

36. Nunes TCM. A supervisão: uma proposta pedagógica para o setor saúde. Cad Saúde Pública. 1986 out-dez; 2(4):466-476.

37. Freese E, Machado E, Cesse E. Analisando situações de governo em municípios pernambucanos: quem busca e quem produz mudanças na gestão do SUS. In: Freese E, organizador. Municípios: a gestão da mudança em saúde. Recife: UFPE; 2004. p. 261296.

38. Alves CKA, Carvalho EF, Cesse EAP, et al. Análise da implantação de um programa com vistas à institucionalização da avaliação em uma Secretaria Estadual de Saúde. Rev Bras Saúde Matern Infant. 2010 nov; 10(supl.1):145-156.

39. Lima EC, Vilasbôas ALQ. Implantação das ações intersetoriais de mobilização social para o controle da dengue na Bahia, Brasil. Cad Saúde Pública. 2011 ago; 27(8):1507-1519.

40. Matus C. El método PES: reingenieria pública y la teoria de las conversaciones: trabas y problemas. Caracas: Fondo Editorial Altadir; 1994.

\footnotetext{
Recebido em 01/07/2018

Aprovado em 19/09/2018

Conflito de interesses: inexistente

Suporte financeiro: não houve
} 